\title{
Differentiation of the Vegetative and Sporogenous Phases of the Actinomycetes
}

\section{Variation in the Actinomyces coelicolor species-group}

\author{
BY DAGNY ERIKSON \\ Agricultural Research Council, Rothamsted Experimental Station, \\ Harpenden, Herts
}

\begin{abstract}
SUMMARY: Variation in the Actinomyces coelicolor species-group comprises loss of pigment and aerial mycelium, and occasionally of agar liquefaction. Stable variants may arise from degenerate, aged, vegetative mycelium, but do not normally do so when the vegetative mycelium is kept in vigorous condition by frequent subcultivation in suitable media. Single spore isolations from the aerial mycelium of typical and of variant colonies show that there may be inherent differences in the sisterspores of the same chain. Thus, in an agar-liquefying strain 3 of 15 spores had lost the power to produce pigment and to liquefy agar; and an atypical colony of the same strain yielded three viable isolates each with a strong tendency towards sectoring, with the ultimate production of a colourless, non-agar-liquefying variant as well as the typical growth. A non-agar-liquefying strain, which by prolonged cultivation in the vegetative phase had lost its power of producing the red-blue indicator pigment, yielded a variant giving rise to sectored colonies with occasional restoration of the blue pigment. Spontaneous occurrence of variants may be detected in certain spores of the aerial mycelium of a well-grown typical colony, although it is more readily seen in the spores of degenerate colonies which have been rendered atypical by artificial methods of cultivation.
\end{abstract}

Much of the confusion existing in the literature concerning the extreme variability of the actinomycetes is due to lack of recognition, or forgetfulness, of their peculiar habits of growth. The ordinary actinomycete colony (Cohnistreptothrix Group I Ørskov, Streptomyces Waksman) is not a colony in the bacterial sense; i.e. it is not an assemblage of disparate cells issuing from one or more similar cells, but the filamentous ramifying extension of the parent cell or cells. Moreover, when mature, it is composed of two phases: the vegetative substratum mycelium, and the aerial sporogenous mycelium. Recent work by von Plotho (1940), Klieneberger-Nobel (1947), and Erikson $(1947 a)$, has confirmed the existence of constitutional differences between these phases, already suggested by the studies of Ørskov (1923). It follows, therefore, that in a discussion of variation, such as the frequent phenomenon of asporogenous sectors produced within a single colony, it is essential to consider the origin of the inoculum in every instance. With very few exceptions, this has not been done by students of this particular problem.

Actinomyces coelicolor was selected as the test organism for this study for the following reasons. (1) Like many other saprophytic actinomycetes, it readily produces asporogenous sectors or discrete sporeless colonies on certain media; (2) it is strikingly pigmented, the range of colours varying with the $\mathrm{pH}$ achieved by the growth of the organism (Oxford, 1946; Cochrane \& Conn, 1947) and therefore (yielding a partial index of metabolic activity; (3) some 
strains are agar-liquefying (Stanier, 1942), which constitutes a third variable character.

The strains examined were isolated from Rothamsted soils, and include some of those (U1010, BCA I) studied by Oxford for pigment production. Some of the agar-liquefying strains described by Stanier were obtained through the courtesy of Dr van Niel. Of these the strain Waksman 3443 (W. 3443) was used for detailed study.

\section{MEDIA AND METHODS}

Simple defined media were employed throughout. Routine plating was done on Czapek's sucrose-nitrate agar. Other media used were the starch-tryptone agar of Waksman, and a substrate containing the Czapek inorganic salts plus ammonium lactate $(1 \%)$ or ammonium acetate $(0.5 \%)$ as the sole source of nitrogen and carbon. All were adjusted to $\mathrm{pH} 7 \cdot 0$. Sterile soil moistened with distilled water was used for maintenance of the strains. The platinum loop was used for mass transfers of vegetative and aerial mycelia. From a densely sporulating colony a loopful may contain an enormous number of viable spores, as is shown by the following experiment.

From a 7-day-old culture on starch tryptone, which enhances sporulation, six different loopfuls of aerial mycelium were taken and suspended in separate $5 \mathrm{ml}$. portions of sterile saline, run out in serial dilutions, and plated. In the $1 / 343,000,000$ dilution one colony appeared on each of five plates, and three colonies on the sixth. Owing to the difficulty of making homogeneous suspensions of the not easily wettable spores (Erikson, 1947 a), this does not necessarily mean that every loopful contained as many million spores as the numbers imply.

For small inocula, finely drawn-out capillary pipettes were used for seeding the plates with droplets of spores; and to the suspending fluid was added $0 \cdot 1 \%$ of a commercial detergent, which had been proved to have no toxic effect on viability. From time to time in the course of the experiments, such droplets were incubated in moist chambers with a drop of nutrient broth, and the number of developing spores observed microscopically. The number varied from 1 to 20 or even more, according to the strain, the age of culture, and medium. Despite vigorous shaking of the suspension, it was sometimes found that conidial chains remained intact. Simultaneous germination of two, three, or more numbers of such chains of varying length was observed in 90 cases, as against 893 instances of germination of detached single cells.

It is clear, therefore, that even with these precautions there can be no guarantee that the colonies on one plate are derived from a similar number of elements. Later, with the kind assistance of Dr de Fonbrune of the Pasteur Institut, Garches, the sister-spores of separate chains were isolated by means of his pneumatic micromanipulator (de Fonbrune, 1937).

\section{RESULTS \\ Vegetative mycelium as inoculum}

In the course of 3 years' continuous subculture and observation of 10 different isolates of $A$. coelicolor, occasional smooth sectors (i.e. apparently devoid of aerial mycelium) were seen many times in colonies on the routine media. Such 
vegetative growth was dissected and streaked across fresh plates, and in the majority of instances typical growth with abundant aerial mycelium occurred, if not in the first, then in the second, subculture. The most striking example was that of a culture of strain U 1010, which on Czapek glucose-nitrate agar produced an abnormal smooth orange growth, resembling that of a proActinomyces. This strain was growing in a flask of sucrose-alanine medium, and failed to produce the soluble blue pigment. When this seemingly pure vegetative growth was streaked on Czapek sucrose-nitrate agar, it grew as characteristic colonies bearing grey-white, and occasional drab fawn sectors of aerial mycelium, and produced a limited amount of blue pigment. Further subculture yielded uniform typical growth. On careful microscopical examination of the original smooth orange growth, however, it was found that there were occasional aerial hyphae, undivided into spores, often no larger than minute buds; a development exactly similar to that which can be seen in many of the pro-actinomycetes (cf. Jensen, 1932).

To secure an inoculum in the purely vegetative phase, the aerial mycelium of cultures of each of the 10 isolates was washed off with phosphate buffer from a 7-day growth on cellophan over starch-tryptone agar, to minimize the effect of initial medium; and then dried on sterile cover-slips 9 days in vacuo, to obtain survival of spores only. The resulting spores were subcultured in nutrient glucose broth, where they fell from the cover-slip to the bottom of the tube and produced vegetative mycelia in 3-5 days. This culture was subcultured in the same medium every few days for 5 months; this constant subculturing in tall tubes containing $10 \mathrm{ml}$. of protein-containing media favours profuse growth which, since it is not allowed to reach the surface, is unable to produce aerial mycelium. Parallel sets of nutrient glucose broth cultures were left unsubcultured for the 5-month period.

Of the 10 frequently subcultured strains in the vegetative phase, seven produced uniform characteristic growth on plating on Czapek nitrate sucrose agar. Strain 14 yielded 24, colonies of which one produced only the yellow precursor to the red insoluble pigment. The progeny of this colony were typical blue and yellow colonies in about equal numbers. Strain $\mathrm{Ph}-\mathrm{C}$ gave 20 small colourless colonies, five large typical blue colonies, and one small purple one, Strain pH-R2 was similar, but four of the small colonies had red pigmented sectors. All bore more or less abundant aerial mycelium. On further subculture none of the variants proved to be stable.

When the 5-month-old cultures were examined, only four of the strains still showed bottom vegetative growth. The others had some surface aerial mycelium. Subcultured on various plate media, each strain produced a high proportion of non-pigmented or yellow to reddish purple colonies, often with sectors and without aerial mycelium. One smooth red colony from strain pH-R (1)b was selected for detailed examination. For the first two subcultures it produced a mixture of typical pigmented sporing colonies and of colourless or reddish sporeless colonies. Sowing from these simultaneously, on the two halves of a plate, each of these two types then bred true on all defined media for over a year. But microscopical observation of almost any of the apparently smooth colonies 
revealed that there were some aerial filaments, usually undivided, just as in the orange growth of $\mathrm{U} \mathbf{1 0 1 0}$ noted above. The loss of the power to produce aerial mycelium was not complete.

This was finally proved by growing the non-sporing variants in sterile soil for 2-4 weeks. Crumbs of the inoculated soil became covered with typical aerial spirals subdivided into spores, and on replating this property was retained. The power of pigment production, however, was not usually restored by passage through soil. Nevertheless, this could not be regarded as entirely lost. Occasional bacterial and fungal contaminants producing respectively an alkaline and an acid $\mathrm{pH}$, induced blue and red coloration of the actinomycete growth in their respective vicinities. The colourless colonies had evidently elaborated the precursor to the pigment, although they were unable by their own metabolic activity to establish the requisite $\mathrm{pH}$ for its manifestation.

The vegetative bottom growth from a variety of other media was also tested for uniformity on the routine solid media. These media, adjusted to $\mathrm{pH} \mathrm{7 \cdot 0}$ contained ammonium phosphate as the source of $\mathrm{N}$, the usual Czapek inorganic salts, and $1 \%$ by weight of each of the following substances: sucrose, glucose, lactose, fructose, maltose, glactose, arabinose, raffinose, xylose; mannitol, sorbitol, dulcitol; salicin, inulin, starch; tartaric, succinic, uric, citric, malic, acetic, lactic acids; cellulose (not weighed) in the form of strips of pure Swedish filter-paper. Xylose, dulcitol, tartrate, and citrate did not support growth. From all media that supported good growth, characteristic uniform colonies were produced on plating. Where the growth was poor, as with arabinose, malate, and acetate, there was a tendency towards sectoring. All strains behaved more or less similarly.

The effect of a high concentration of $\mathrm{Ca}$ ion $\left(\mathrm{CaCl}_{2}, 25 \mathrm{~g}\right.$./l.) was also tested in the nitrate-sucrose medium recommended by Foster, McDaniel, Woodruff $\&$ Stokes (1945) for the production of fruiting bodies in submerged cultures of $\boldsymbol{P}$. notatum. In this substrate growth was poor, and the filaments showed an abnormal degree of condensation of the protoplasm in densely staining blocks, as well as thickening of portions of the cell walls. Yet on plating on starchtryptone agar typical colonies were obtained.

\section{Aerial mycelium as inoculum}

Commencing with the same material as was used for the experiments in liquid media, namely, dried washed spores on cover-slips, plates were seeded with droplets of the spore suspension and subcultured regularly during the 5-month period. The same general phenomena were observed. The dominant colony was that characteristic of the species: it bore spirally coiled aerial mycelium abundantly divided into spores, produced a red insoluble pigment in the vegetative mycelium and a blue soluble pigment that diffused into the medium as it became alkaline. From time to time many variants were observed on the plate; colourless, pale pink, red, purple, with yellow, orange or green reverse; varying greatly in size, and having no aerial mycelium or very little, mostly not divided into spores. On subculture these variants 
frequently produced similar mixtures, but also some of the dominant colonies. Mass transfers of typical growth usually yielded uniform growth on plates.

\section{Monoconidial isolates}

Micromanipulator isolations were made from four different types of colony, two each from two different strains :

(1) A typical well-sporulated, vigorous, blue-pigmented, agar-liquefying, colony of W. 3443.

(2) A poorly sporulated, yellow-pigmented, sectored, non-agar-liquefying colony of W. 3443; of the same age, and on the same medium as (1).

(3) A well-sporulated, vigorous, yellow-pigmented colony of the variant Ph-R (1) $b$, which for a year had been kept almost entirely sporeless and colourless, but which after passage through sterile soil had recovered the power of producing aerial mycelium, and to a slight extent that of pigment production.

(4) A poorly sporulated, colourless, sectored colony of $\mathrm{Ph}-\mathrm{R}(1) b$, of the same age, and on the same medium as (3).

The spores were incubated in droplets of nutrient glucose broth in the oil chamber of Comandon \& de Fonbrune (1938). Not all were viable. There were also observable differences in the rate of germination. From (1), three chains, $1 / 1,1 / 2,1 / 3$, were isolated and the sister-spores separated and cultured simultaneously under identical conditions:

1/1: six spores; two $(a$ and $b$ ) viable;

$1 / 2$ : eight spores; eight $(a-h)$ viable;

$1 / 3$ : eight spores; five $(a-e)$ viable.

When seeded on Czapek nitrate-sucrose agar for ten generations, the following results were obtained:

1/1 a: uniform characteristic growth;

$1 / 1 b$ : uniform growth, but only yellow pigment, no agar liquefaction;

$1 / 2 a$ : uniform characteristic growth;

$1 / 2 b$ : a colourless, non-agar-liquefying variant obtained, breeding true for 30 generations (Pl. 1, fig. 1-3);

$1 / 2 c, 1 / 2 d, 1 / 2 e, 1 / f$ : all uniform characteristic growth;

$1 / 2 g$ : a variant similar to $1 / 2 b$;

$1 / 2 h$ : uniform characteristic growth;

$1 / 3 a-1 / 3 e$ : all five spores yielded uniform characteristic growth.

Thus, of the 15 strains derived from spores taken from three separate chains from a well-grown colony, 12 produced the characteristic growth, and three had lost the power to produce pigment and to liquefy agar.

From colony 2, two chains were isolated:

$2 / 1$ : twelve spores; two $(a$ and $b$ ) were viable;

2/2: eight spores; one $(a)$ was viable.

All three isolates tended strongly to grow in sectored colonies with loss of pigmentation, incomplete sporulation, and inability to liquefy agar. Ultimately, two separate variants, corresponding to those shown in $\mathrm{Pl}$. 1, fig. 1, were obtained from 2/1a. 
From colony 3, two chains were isolated:

3/1: eight spores; $\operatorname{six}(a-f)$ were viable;

3/2 : eight spores; eight $(a-h)$ were viable.

Only one, $3 / 2 f$, of the 14 single-spore strains varied, producing colourless, sporeless, and blue-pigmented sectors. The rest produced good aerial mycelium and varying degrees of yellow pigmentation on the reverse of the colony, as in the parent culture.

From colony 4, two chains were isolated:

$4 / 1$ : eight spores; four $(a-d)$ were viable.

4/2: four spores; one was viable.

All five isolates yielded poor growths with a marked tendency towards sectoring; colourless, with sparse aerial mycelium; with yellow to reddish pigment, and irregular aerial mycelium. In no instance was the typical, wellsporulated, blue-pigmented colony obtained.

\section{Sector production}

The suggestion that multiple sectored colonies arise from more than one conidium, which has frequently been made (Stanier, 1942), has not been substantiated. This is shown in Pl. 1, fig. 4; $A$ was seeded with the colourless, non-agar-liquefying variant $1 / 2 b$, and $B$ with a culture typical of the parent strain W.3443, both derived from the same single spore. The central portion was seeded with a mixture of spores from $1 / 2 b$ and the typical growth. The appearance of the mixed colonies was the same as that of the typical growth, as regards aerial mycelium, pigment production, and a slight degree of agar liquefaction. Multiple colonies could be seen, but they were uniform, not sectored. Further experiments were made, growing $1 / 2 b$ and the typical strain separately and mixed, in liquid media and in soil, and then plating both mixed suspensions of spores from the separate growths and suspensions of spores from the mixed growth; the resultant colonies could not be distinguished from the dominant typical colonies.

\section{DISCUSSION}

If we disregard all the temporary variants manifested by the different shades of pigmentation and varyingly efficient degrees of sporulation, the main variable characters to be considered are pigment, aerial mycelium and agar liquefaction.

Complete loss of pigmentation was maintained during 1 year's frequent subculturing with strain $\mathrm{Ph}-\mathbf{R}(\mathbf{1}) b$, derived from the vegetative mycelium of a 5-month-old broth culture. Simultaneously, the power of producing aerial mycelium was so very greatly diminished, that to the naked eye the mycelium appeared to be sporeless.

Vegetative mycelium in a vigorously growing state produced only occasional variants with temporary loss of these two attributes, whatever the liquid medium in which it was grown. It is clear, therefore, that it is the degraded condition and diminution of vigour induced by artificial means which bring 
about the variation by loss. This is confirmed by microscopical examination of such aged mycelium, which shows an extraordinary degree of beading, segmentation, and vacuolation of the filaments, and only very small portions of which proved to be viable; and by the fact that passage in soil, a natural habitat, restores the property of producing aerial mycelium, and to a slight extent that of pigmentation. Normally, then, the vegetative mycelium reproduces its kind.

Analysing the results of culturing the single spores of two different types of colony in two separate strains, we find that a higher proportion of spores were viable and their progeny more stable in the case of the two vigorous, wellsporulated colonies : 1 (W.3443), and $3(\mathrm{Ph}-\mathrm{R}(1) b)$. The less well-developed parent colonies, $2(W .34,43)$ and $4(\operatorname{Ph}-R(1) b)$, which already showed diminution in aerial mycelium production and little or no pigmentation, yielded a lower proportion of viable spores whose progeny was marked by sectoring and instability. This accords with expectation, but in the time available the numbers of single spores isolated were not large.

Nevertheless, variation can occur within a homogeneous population in a vigorous state of growth. Thus, in the variant $1 / 2 b$, the typical pigmented, agar-liquefying colonies on one half of $\mathrm{Pl}$. 1, fig. 1 are strikingly different from the equally vigorous, even larger, colourless, non-agar-liquefying colonies on the other half. Yet all are the progeny of one spore. There is also a difference in the degrees of brittleness and non-wettability of the aerial mycelium in each case, the non-pigmented variety being more easily detached and considerably more resistant to the action of wetting agents (see Erikson, 1947 b; also Velu, Comandon, de Fonbrune, Janot, Penau, Mainil \& Bouet, 1947, on monoconidial isolates of $\boldsymbol{P}$. notatum). Six of the sister-spores of the same chain produced only the typical growth. One must conclude that there is an inherent tendency for a given mutation to occur more frequently in one clone than another.

The linkage of pigmentation with agar-liquefaction is not quite complete. Passage of the colourless $1 / 2 b$ in soil restored a very slight degree of pigmentation (occasional yellow-green patches), but not that of agar-liquefaction. As Braun (1947) has pointed out, many linked characters can be attributed to one basic change only; or to a single mutation at different levels of gene-controlled processes; or to the influence of one mutation on the rate of occurrence of another mutation. The mechanism is not yet clear.

The tendency of some spores to produce colourless variants is commonly masked by the pigmentation of the dominant parent strain, as is shown by the experiments with the mixture of spores from the two types of $1 / 2 b$. Schaal (1944) also has illustrations of colourless and pigmented variants from a single spore of Actinomyces scabies.

This work was carried out by the author as a member of the scientific staff of the Agricultural Research Council.

I wish to thank Dr H. G. Thornton, F.R.S., for suggestions, and, together with Mr Stansfield, for taking the photomicrographs; Dr P. de Fonbrune for hospitality, instruction, and assistance in making the micromanipulator isolations; and Miss Enid Wilsher for technical assistance. 
Journal of General Microbiology, Vol. 2, No. 3
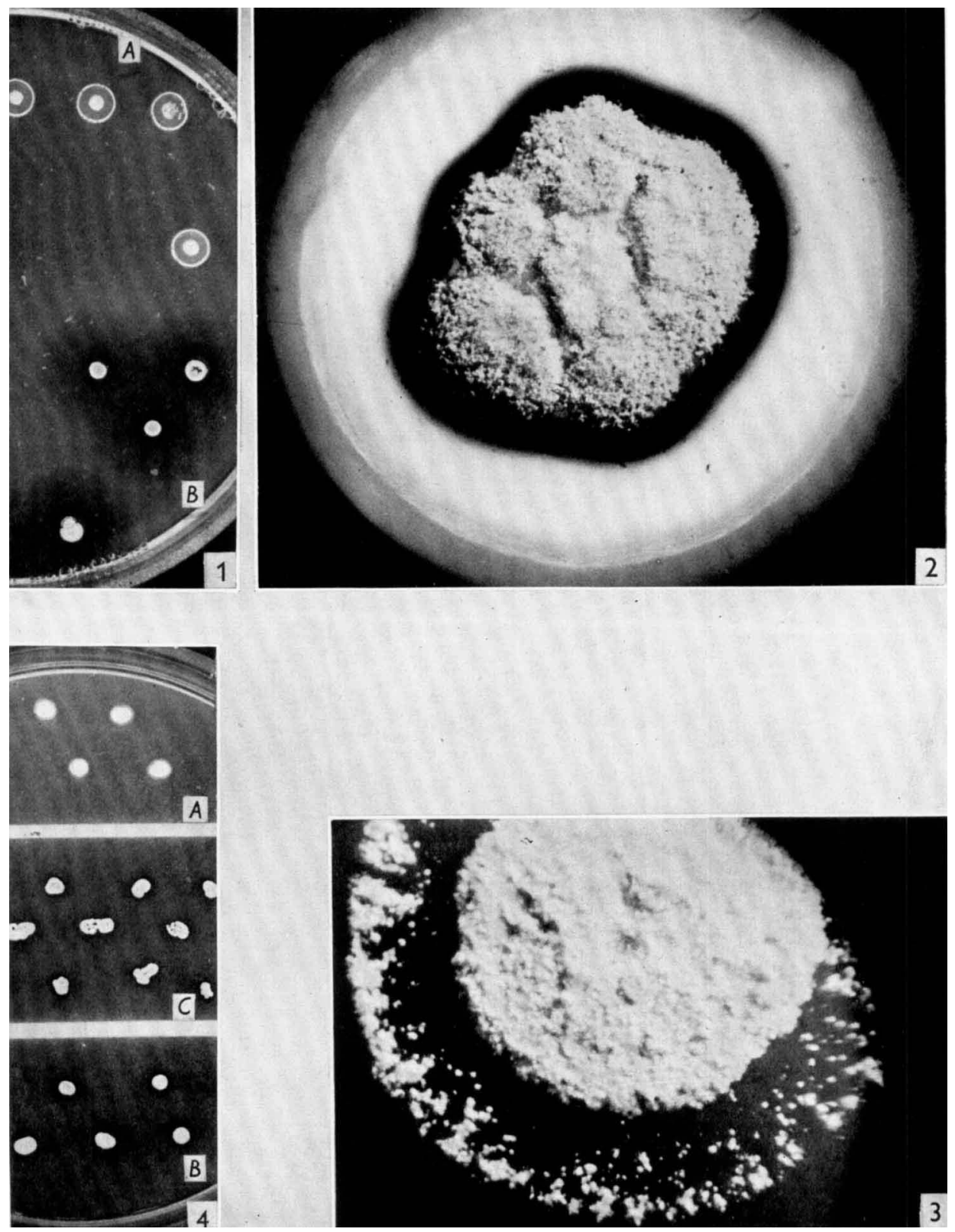

Figs. 1-4

D. Erikson-Variation in $A$. Coelicolor. Plate 1 


\section{REFERENCES}

BraUn, W. (1947). Bacterial dissociation. Bact. Rev. 11, 75.

Cochrane, V. W. \& Conn, J. E. (1947). The growth and pigmentation of $\boldsymbol{A}$. coelicolor as affected by cultural conditions. J. Bact. 54, 213.

Comandon, J. \& Fonbrune, P. de (1938). La chambre à huile. Ses avantages pour l'étude des microorganismes vivants, la culture des tissus et la micromanipulation. Ann. Inst. Pasteur, 60, 113.

Erikson, D. (1947 a) Differentiation of the vegetative and sporogenous phases of the actinomycetes. I. The lipid nature of the outer wall of the aerial mycelium. J. gen. Microbiol. $1,39$.

ErIKson, D. (1947b). Differentiation of the vegetative and sporogenous phases of the actinomycetes. II. Factors affecting the development of the aerial mycelium. J. gen. Microbiol. $1,45$.

Fonbrune, P. DE (1937). Micromanipulateur pneumatique et Micro-Forge. Paris: Société industrielle d'Imprimerie.

Foster, F. W., McDanier, L. E., Woodruff, H. B. \& Stokes, J. L. (1945). Conidiospore formation in submerged cultures of $P$. notatum. J. Bact. 50, 365 .

Jensen, H. L. (1932). Contributions to our knowledge of the Actinomycetales. IV. The identity of certain species of Mycobacterium and Proactinomyces. Proc. Linn. Soc. N.S.W. 57, 364.

KLIENEBerger-Nobel, E. (1947). The life cycle of sporing Actinomyces as revealed by a study of their structure and septation. J. gen. Microbiol. 1, 22.

ØRskov, J. (1923). Investigations into the Morphology of the Ray Fungi. Copenhagen: Levin and Munksgaard.

OXFord, A. E. (1946). Note on the production of soluble blue pigment in simple media by Actinomyces coelicolor. J. Bact. 51, 267.

Plotho, O. von (1940). Die chromatische Substanz bei Actinomyceten. Arch. Mikrobiol. 11, 285.

ScHAAL, L. A. (1944). Variation and physiologic specialization in the common scab fungus (Actinomyces scabies). J. Agric. Res. 69, 169.

Stanier, R. Y. (1942). Agar-decomposing strains of the Actinomyces coelicolor speciesgroup. J. Bact. 44, 555.

Velu, H., Comandon, J., Fonbrune, P. de, Janot, M., Penau, H., Mainil, J. \& Bouet, G. (1947). Essais de dissociation et de sélection de Penicillium notatum. Ann. Inst. Pasteur, 73, 1.

\section{EXPLANATION OF PLATE}

Fig. 1 . The two variants of $1 / 2 b$ derived from one spore; $(A)$ colourless, non-agar-liquefying; $(B)$ blue-pigmented, agar-liquefying.

Fig. 2. Discrete colony from $(B)$ in Fig. 4; the aerial mycelium is colourless, the substratum mycelium is red, and a ring of soluble blue pigment has diffused into medium. $\times 16$.

Fig. 3. Part of a discrete colony from $(A)$ in Fig. 4; showing different disposition of colourless aerial mycelium.

Fig. 4. The two variants of $1 / 2 b,(A)$ and $(B)$ shown in Fig. 1 , and $(C)$ a mixture of the two. 